Anaesthesist 2015 - 64:345-346

DOI 10.1007/s00101-015-0041-7

Online publiziert: 19. Mai 2015

(c) Springer-Verlag Berlin Heidelberg 2015

\section{T. Standl}

Klinik für Anästhesie, Operative Intensiv- und Palliativmedizin, Städtisches Klinikum Solingen gGmbH, Akademisches Lehrkrankenhaus der Universität zu Köln, Solingen, Deutschland

\title{
Niemandem schaden
}

„Primum non nocere“ oder auch „Primum nihil nocere" lautet der bis zum heutigen Tag geltende Grundsatz in der Medizin, der sich aus dem hippokratischen Eid herleitet. Er wird in seiner lateinischen Version dem Arzt Scribonius Largus zugeschrieben, der am Hof des Kaisers Tiberius Claudius etwa 50 n. Chr. gewirkt hat. Dieses Prinzip, dem Patienten mit der ärztlichen Therapie zumindest nicht zu schaden, zieht sich bis in die heutige Zeit hinein und hat gerade im Bereich der Geburtshilfe besondere Bedeutung.

Bei der schwangeren Patientin und stillenden Mutter geht es bekanntermaBen nicht nur um die Gesundheit der (werdenden) Mutter, sondern insbesondere auch um die ungestörte Entwicklung des Embryos bzw. Fetus sowie die Vermeidung von negativen Einflüssen auf das Neugeborene und den Säugling. Auch die Sorge des Anästhesisten gilt bei schwangeren Patientinnen und in der peripartalen Phase mindestens 2 Patienten: der werdenden oder gerade gewordenen Mutter und dem Kind. Diese grundsätzliche Haltung, die v. a. eine Aufrechterhaltung der Homöostase der Mutter beinhaltet, bestimmt in allen Phasen der Schwangerschaft und Entbindung unser anästhesiologisches Tun. Das bedeutet einerseits eine Auswahl besonders schonender und nebenwirkungsarmer Verfahren (Regionalanästhesie vs. Allgemeinanästhesie) sowie und insbesondere die Auswahl von Medikamenten, die nicht teratogen sind und keine negativen Auswirkungen z. B. auf die uteroplazentare Perfusion oder nach der Geburt auf die Atemfunktion des Neugeborenen haben [1]. Die Durchführung von anästhesiologischen Techniken und Verfahren sowie der Schmerztherapie ist genuiner Bestandteil der Tätigkeiten des Anästhesisten. Hierbei haben das Wissen um eine mögliche teratogene Nebenwirkung von Pharmaka und die Vermeidung der Anwendung dieser Medikamente im 1. Trimenon herausragende Bedeutung [2]. In diesem Zusammenhang soll an die katastrophalen Auswirkungen des - allerdings nicht durch Anästhesisten verschriebenen Medikaments - Thalidomid $\left(\right.$ Contergan $\left.^{\circ}\right)$ in der Zeit um 1960 erinnert werden, das schwerste Fehlbildungen im Bereich der Extremitäten und inneren Organe bei Neugeborenen zur Folge hatte, obwohl Tierversuche kein teratogenes Potenzial befürchten ließen. Die Aufarbeitung dieses Skandals hat zu einer sehr genauen Vorstellung über die Phasen der Organogenese des menschlichen Embryos und über den jeweiligen Zeitpunkt der Schädigung durch Thalidomid geführt [3]. Andere Befürchtungen, wie z. B. eine vermehrte Inzidenz von Lippen-Kiefer-Gaumenspalten nach Einnahme von Benzodiazepinen während der Schwangerschaft konnten wissenschaftlich nicht belegt werden [4].

Für den Anästhesisten ist es wichtig, ein Repertoire an Medikamenten zur Hand zu haben, das im 1. Trimenon zur Versorgung der Schwangeren z. B. im Rahmen von Notfällen (Frakturen, akutes Abdomen, Thyreotoxikose u. a.) sicher zur Anästhesie und zur Schmerztherapie eingesetzt werden kann. Ein schwieriger Punkt bleibt die Indikation von Analgetika z. B. bei Patientinnen mit chronischem Schmerz. Auch hier dürfen im 1. Trimenon keine toxischen oder teratogen wirksamen Substanzen eingesetzt werden. Im letzten Trimenon und präpartal gilt die Sorge insbesondere möglichen atemdepressiven Nebenwirkungen (Opioide) oder speziellen Kontraindikationen wie z. B. nichtsteroidalen Antirheumatika (NSAR) und dem vorzeitigen Verschluss des Ductus arteriosus beim Neugeborenen.
Es ist das Verdienst von Ninke et al. in dieser Ausgabe von Der Anaesthesist, dieses komplexe Thema in strukturierter und übersichtlicher Form zu präsentieren und für den klinischen Alltag klare Empfehlungen für diese spezielle $\mathrm{Pa}$ tientengruppe zu geben [5]. Besonders wichtig erscheint in diesem Zusammenhang der Hinweis auf die Homepage der Datenbank „embryotox“, die eine sehr umfangreiche Zusammenstellung von Pharmaka und ihrer möglichen Auswirkungen im Rahmen der Schwangerschaft darstellt [6].

Die anästhesiologische Betreuung einer schwangeren Patientin und werdenden Mutter stellt nach wie vor hohe Ansprüche und eine gewisse Herausforderung an alle Beteiligten im Arzt- und Pflegedienst dar, zumal auch in unserem Land in den letzten Jahren der Anteil an Risikoschwangerschaften und -geburten kontinuierlich zugenommen hat [7-9]. Insofern ist die nachfolgende Publikation ein begrüßenswerter Beitrag zu einer wichtigen Thematik mit klinischer Relevanz.

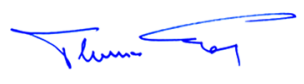

\section{T. Standl}

\section{Korrespondenzadresse}

\section{Prof. Dr. T. Standl}

Klinik für Anästhesie

Operative Intensiv- und Palliativmedizin

Städtisches Klinikum Solingen gGmbH

Akademisches Lehrkrankenhaus der

Universität zu Köln, Gotenstr. 1

42653 Solingen

standl@klinikumsolingen.de

\section{Einhaltung ethischer Richtlinien}

Interessenkonflikt. T. Standl gibt an, dass kein Interessenkonflikt besteht. 


\section{Literatur}

1. Girard T, Brugger S, Hösli I (2013) New aspects of obstetric anesthesia. Anaesthesist 62(12):963-972

2. Paulus WE (1999) Pharmacotherapy in pregnancy. Ther Umsch 56(10):602-607

3. Ridings JE (2013) The thalidomide disaster, lessons from the past. Methods Mol Biol 947:575-586

4. Shiono PH, Mills JL (1984) Oral clefts and diazepam use during pregnancy. N Engl J Med 311(14):919920

5. Ninke T, Thoma-Jennerwein S, Blunk J, Annecke T (2014) Anesthesia and pain management during pregnancy. Anaesthesist. doi:10.1007/s00101-0150023-9

6. www.embrytox.de. Arzneimittelsicherheit in Schwangerschaft und Stillzeit. Informationsseite des Pharmakovigilanz- und Beratungszentrums für Embryonaltoxikologie

7. Kramer MS, Berg C, Abenhaim H et al (2013) Incidence, risk factors, and temporal trends in severe postpartum hemorrhage. Am J Obstet Gynecol 209(5):449.e1-e7

8. Schlembach D, Mörtl MG, Girard T et al (2014) Management of postpartum hemorrhage (PPH): algorithm of the interdisciplinary D-A-CH consensus group PPH (Germany - Austria - Switzerland). Anaesthesist 63(3):234-242

9. Löser B, Tank S, Hillebrand G et al (2013) Peripartum cardiomyopathy: interdisciplinary challenge. Anaesthesist 62(5):343-354

\section{St. Pierre, G. Hofinger}

\section{Human Factors und Patientensicherheit in der Akutmedizin}

Heidelberg: Springer Verlag 2014, 3. überarb. Auflage, 368 S., 62 Abb., (ISBN 978-3662-45879-2), 59.99 EUR

Das in den ersten beiden Auflagen unter dem Titel „Notfallmanagement" erschienene Buch ist in der 3. Auflage nochmals deutlich erweitert und komplett aktualisiert. Es zählt hiermit weiterhin zu den herausragenden Standardwerken mit allen wesentlichen Aspekten des modernen akutklinischen Risikomanagements und der Patientensicherheit. Die neue Auflage besticht durch eine noch größere Praxisnähe zur klinischen Routinearbeit, indem viele sorgfältig erprobte Umsetzungskonzepte aufgenommen und um aktuelle Themenkomplexe erweitert wurden. Insbesondere der neu konzipierte und stark erweiterte Überblick über das The-menfeld "Organisation" spiegelt umfassend die neuesten Ergebnisse aus der Organisationspsychologie, den aktuellen Erkenntnissen des Risikomanagements und der klinischen Arbeit wider.

Das Werk fokussiert sehr gut auf die wesentlichen Mechanismen von akutmedizinischen Entscheidungsfindungen inklusive der hierin impliziten - nicht selten erfolgskritischen - Human Factor Aspekte. Es beschreibt tief- greifend und verständlich die zentralen (Klinik-)Teamaspekte mit den korrespondierenden organisatorischen und psychologischen Rahmenbedingungen.

Das Buch zeigt eindrucksvoll - in einer sehr klaren Sprache und vielen anschaulichen Darstellungen - den aktuellen Erkenntnisstand hinsichtlich des Zusammenwirkens differenter akutklinischer Systemebenen und ihren direkten oder mittelbaren Einfluss auf konkretes menschliches Handeln im klinischen Alltag. Es verfügt über eine exzellente aktuelle Literaturübersicht, ein durchdachtes Stichwortregister und viele Gliederungshilfen zum schnellen Memorieren und Wiederauffinden.

Das Buch gibt allen in der Akutmedizin arbeitenden Menschen, berufs- und disziplinübergreifend, konkrete Unterstützung und ermöglicht tiefgreifendes Verständnis von aktuellen Erkenntnissen zur Patientensicherheit und den prognosebestimmenden Faktoren im klinischen Risikomanagement. Es wird für Berufsanfänger und für Erfahrene gleichermaßen wertvolle Handlungsorientierung im täglichen klinischen Alltag liefern. Dieses Buch verdient einen festen Platz am Arbeitsplatz jeden Entscheidungsträgers in der Akutmedizin

München/ Hamburg

Prof.Dr.C.K. Lackner/Prof.Dr.Hp.Moecke/ Dr.phil.Dipl.-Psych.K.Burghofer 